\title{
Evaporation of Binary Mixtures and Precision Measurement by Crystal Resonator
}

\author{
Hanyu Song, ${ }^{1}$ Chi-Ruei He, ${ }^{2}$ Carl Basdeo,${ }^{1}$ Ji-Qin Li,${ }^{1}$ Dezhuang \\ Ye, ${ }^{1}$ Devendra Kalonia, ${ }^{3}$ Si-Yu Li, ${ }^{2}$ and Tai-Hsi Fan ${ }^{*}, 1$ \\ ${ }^{1}$ Mechanical Engineering, University of Connecticut, Storrs, CT 06269-3139, USA \\ ${ }^{2}$ Chemical Engineering, National Chung Hsing University, Taichung 402, Taiwan \\ ${ }^{3}$ School of Pharmacy, University of Connecticut, Storrs, CT 06269-3139, USA
}

(Dated: April 23, 2016)

Theoretical and experimental investigations are presented for the precision measurement of evaporation kinetics of binary mixtures using a quartz crystal resonator. A thin layer of light alcohol mixture including a volatile (methanol) and a much less volatile (1-butanol) components is deployed on top of a crystal resonator for the evaporation experiment. A one-dimensional theoretical model is developed to describe the underlying mass transfer and interfacial transport phenomena. Along with the theoretical analysis, the transient evaporation kinetics, moving interface, and the stratification of viscosity of the liquid mixture during evaporation can be simultaneously measured by the impedance response of the shear and longitudinal waves emitted from the resonator. The result on the binary mixture presents a simplified model system for further investigations of complicated evaporation kinetics involving complex fluids or multi-component fuel systems.

Keywords: Evaporation, binary mixture, moving interface, diffusion, crystal resonator

\footnotetext{
* Corresponding author. E-mail: thfan@engr.uconn.edu
} 


\section{INTRODUCTION}

Quantitative analysis of evaporation kinetics of binary or multi-component fluid mixtures is important in many applications ranging from petroleum, transportation, pharmaceutical, to biotechnology industry. Properties of the multi-component fluids are determined by the composition and concentration of the base fluids. Their dynamic behaviors also depend on the transient evolution of the base fluids involved in the process. Thereby better understanding of evaporation kinetics is important in designing, controlling, and optimizing the relevant chemical processes. Evaporation of small liquid drops can be measured by atomic force microscope cantilevers [1] with high sensitivity and time resolution and with the surface tension effect taken into account. For a simpler experiment, it is known that quartz crystal microbalance (QCM) or resonator along with dissipation (QCM-D) and electrochemical (E-QCM) methods have been successfully applied to ultrasensitive mass detection, thin film thickness and rigidity detection, viscosity measurement, and sensing of various interfacial molecular activities, e.g. [2-6]. A few investigations have shown that evaporation kinetics of pure [7-9] and complex fluids including suspensions [10, 11] and polymer solutions [12] can also be measured by crystal resonators. However, theoretical modeling and data interpretation on multi-component systems are only at the beginning stage. The full potential of quartz crystal resonator as a low cost, repeatable, highly sensitive, multi-mode and in-situ sensor for characterizing simple and complex fluids is largely unexplored.

A thin film deposited on top of the surface of an oscillatory crystal will lead to a reduction of the resonant frequency of the whole system [13]. This is because the shear wave propagated into the film deposition increases the effective thickness of the crystal. Similarly, a sessile liquid droplet or liquid layer loaded on top of a crystal resonator will also lead to a change of frequency response [14]. For Newtonian fluids, Kanazawa and Gordon first developed a physics-based model to connect the frequency shift of the resonator to the fluid properties [15, 16]. Their result has been broadly applied to viscosity measurement, which only requires a small amount (less than $1 \mathrm{~mL}$ ) of liquid samples to perform the precision experiment. In addition to the oscillatory shear motion, crystal resonator also oscillates in the normal direction simultaneously and produces a longitudinal acoustic wave propagating toward the liquid-vapor interface [17]. The acoustic wave partially transmits and reflects at the liquid- 


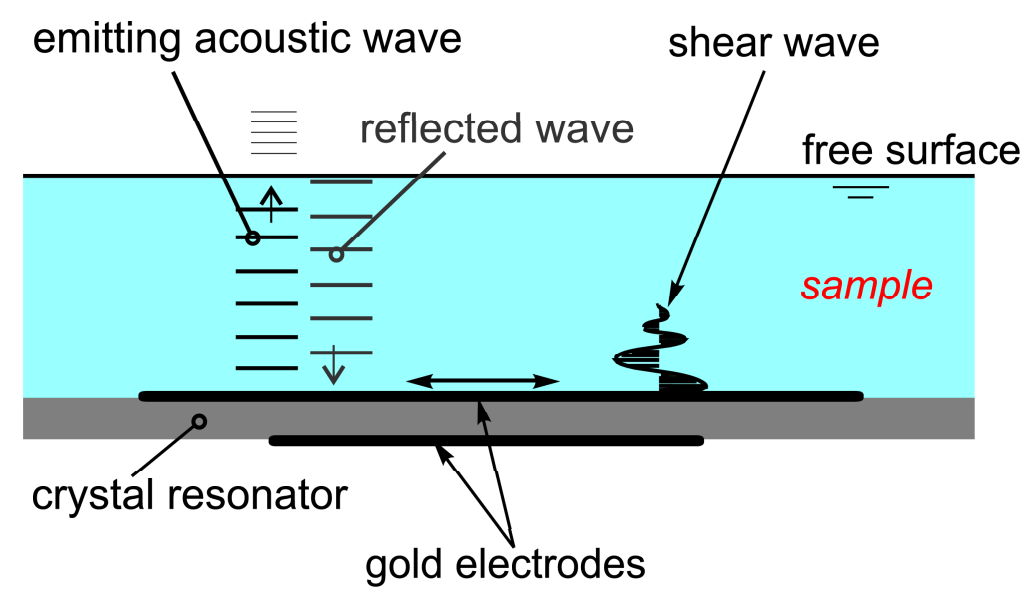

FIG. 1: Schematic showing a liquid layer deployed on top of a quartz crystal resonator, the shear wave near the crystal surface, the longitudinal acoustic wave that penetrates through the liquid layer and is partially reflected by the free surface. The resonator is made of piezoelectric material with electrodes coated on top and bottom surfaces.

vapor interface. The reflected wave again propagates through the liquid layer (Fig. 1) and causes resonance in the liquid while the thickness of the liquid layer evolves to a multiple of half wave length [18]. Each resonance will create a spike response on the mechanical impedance to the crystal, essentially a jump of hydrodynamic resistance to the crystal's motion. Therefore, a transient evaporation on top of the resonator will generate a sequence of spikes (Fig. 2) that one can use to extract the time evolution of the moving liquid-vapor interface with spatial resolution down to several microns. The speed of the moving interface represents the mass flux and directly characterizes the evaporation kinetics.

Here we apply the crystal resonator at the megahertz frequency for the precision measurement of evaporation kinetics of binary (methanol and 1-butanol) fluids. These light, monohydric alcohols are selected as a simplified model system because of their distinct volatility and similar molecular structure for an ideal solution behavior. The evaporation kinetics is measured by the resonant pattern of the acoustic wave (Fig. 2), while the viscosity stratification in liquid mixtures during the evaporation process is evaluated by the shear wave near the surface. Figure 2 shows a typical impedance response of the resonator in terms of the shift of the resonant frequency versus time, demonstrating that methanol evaporates much faster than 1-butanol, and as expected the evaporation rate of a mixture of methanol-butanol is in between pure methanol and pure butanol. Time evolution between 
spikes indicates a reduction of the thickness of the liquid layer by a half of acoustic wave length, around tens of microns. The resonant frequency shift along the base of the signal line is connected to the change of local density and viscosity of the mixture. By tracing the impedance or frequency response and applying the multi-phase modeling results, the evaporation kinetics and transient composition of the liquid layer can be resolved simultaneously.

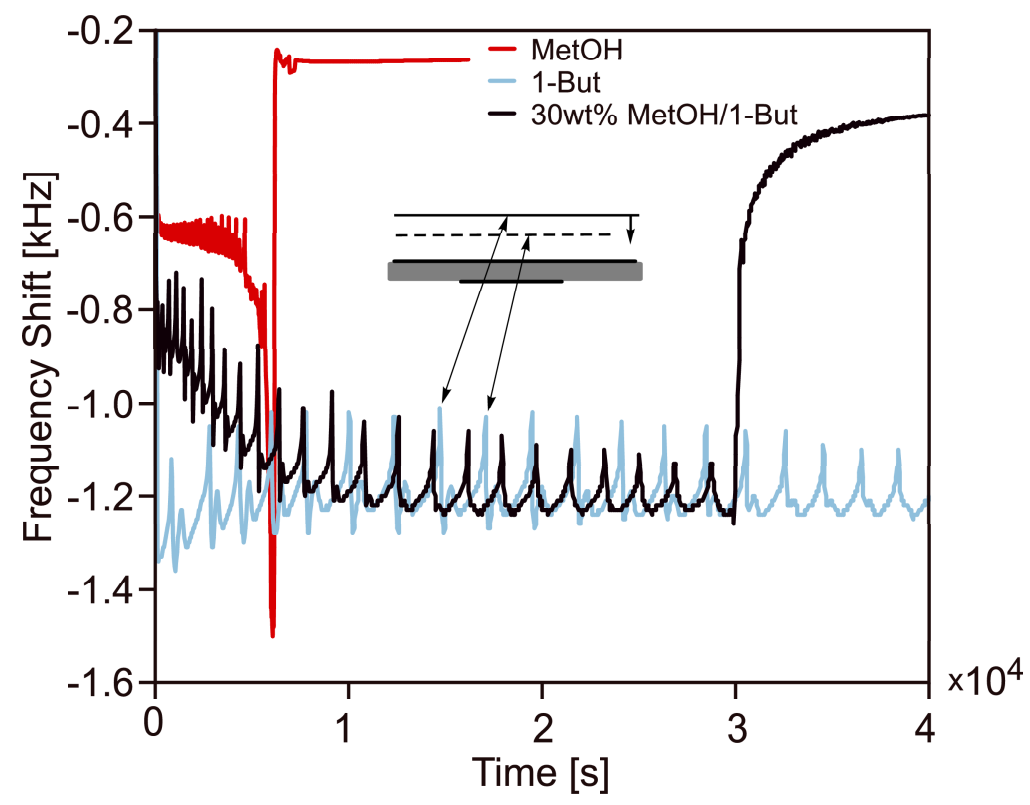

FIG. 2: A typical impedance response in terms of frequency shift of the resonator versus time during the evaporation process. Samples applied are pure methanol, pure 1-butanol, and methanol (30\%)-butanol mixture. Figure inset indicates the free surface locations that correspond to two consecutive resonant spikes.

From modeling perspective, an isothermal evaporation process driven by vapor diffusion from a free surface is categorized as the Stefan problem $[19,20]$. The analytical result for cases with stationary phase change interface was developed by Lee et. al. [21] and later by Prata et al. [22]. Approximation of more complicated isothermal evaporation of a pure and ideal binary mixtures that involve a moving interface was first proposed by Slattery and Mhetar $[23,24]$. In their model for the binary mixture, the interface concentration is presumed fixed to facilitate the analytical solution. The diffusion-induced bulk flow was resolved by Chebbi and Selim [25]. A similar binary problem but with multiple gas components, in which the Stefan-Maxwell equation is applied to describe multi-component diffusion, was investigated by Carty and Schrodt [26]. An ideal binary mixtures may consist of a volatile 
and a non-volatile liquids with or without density difference [27]. To facilitate the analytical approximation, a simplified boundary condition to define the concentration at the liquidvapor interface is usually assumed. A more realistic interfacial condition for an ideal mixture is to take concentration partition into account based on the Raoult's law, i.e., a transient jump concentration across the interface. This interfacial condition plays an important role in characterizing evaporation kinetics of multi-component liquids. Here a one-dimensional numerical model and analytical approximations are developed to predict the concentration field, the change of local viscosity, the moving interface, and the evaporation kinetics of the mixture. The models connect the underlying mass and momentum transport principles to the experimentally observed impedance response for data interpretation.

\section{THEORETICAL ANALYSIS}

A few assumptions are proposed next to simplify the theoretical analysis. A thin layer of methanol-butanol liquid mixture is deployed on top of the crystal resonator. The bare crystal has resonant frequency about $5 \mathrm{MHz}$. The thickness of the liquid layer is around 1 to $4 \mathrm{~mm}$, much less than the diameter of the crystal around $2.4 \mathrm{~cm}$, and thus the onedimensional approximation is appropriate for the problem in hand. Figure 1 shows the schematic of the 1D model. Because the saturation pressure of methanol, $\sim 16.9 \mathrm{kPa}$ under ambient conditions [28], is much higher than that of the 1-butanol, $\sim 0.9 \mathrm{kPa}$ [29], here only methanol is considered volatile and exists in the gas phase such that the multi-component (methanol, 1-butanol, and air) system is very much simplified to a binary mixture of air and methanol. The system is assumed to be isothermal. Evaporation cooling and natural convection are neglected, and the system is approximated to be one dimensional. Both air-methanol gas mixture and methanol-butanol liquid mixture are assumed ideal. The evaporation process starts from a well-mixed liquid layer and only air initially exists in the gas phase. During the process, the thickness of the liquid layer reduces until the methanol is almost depleted. The nonlinear model with moving liquid-vapor interface is simplified and computed numerically, and validated by short- and long-time analytical approximations. 


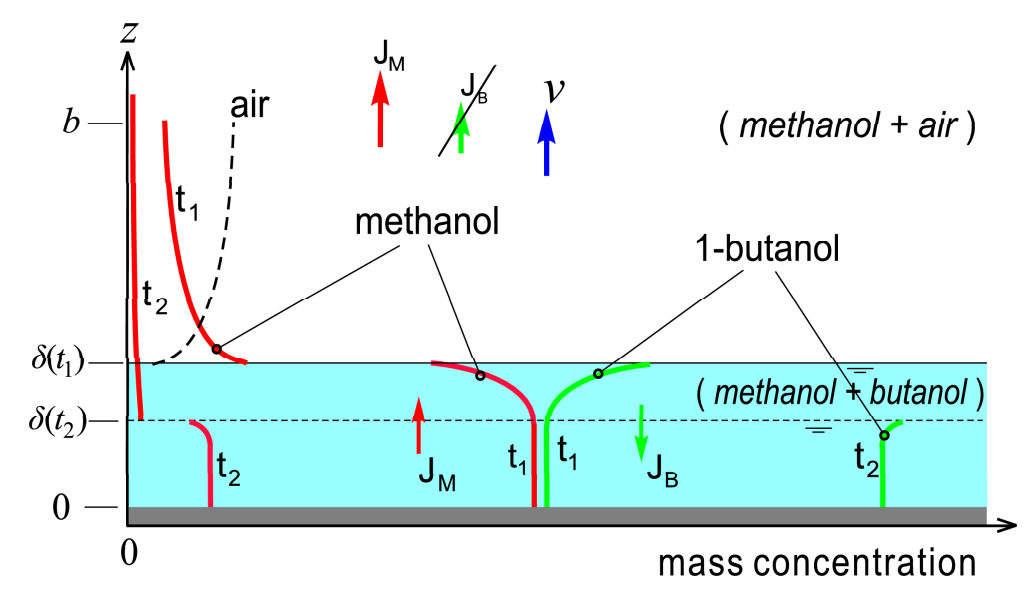

FIG. 3: Schematic of the 1D evaporation of methanol-butanol mixture with assumed equal amount of methanol and 1-butanol initially. The spatial coordinate origin is located on top of the crystal surface. Time instances $t_{1}$ and $t_{2}$ indicate transient evolution of the concentration profiles, and $\delta$ is the thickness of the whole liquid layer, which also indicates the location of the free surface moving toward the crystal surface. $\boldsymbol{J}_{\mathrm{M}}$ and $\boldsymbol{J}_{\mathrm{B}}$ are diffusive fluxes of methanol and butanol, respectively. $\boldsymbol{J}_{\mathrm{B}}$ in vapor phase is assumed negligible. The velocity $v$ is for the uniform upward draft everywhere in the vapor phase.

\section{A. Mathematical Formulations}

The transient mass concentration of the binary mixtures can be obtained by tracking the methanol concentration. In the liquid phase, the mass concentration is governed by

$$
\frac{\partial \rho_{\mathrm{M}}^{(\ell)}(z, t)}{\partial t}=-\nabla \cdot \boldsymbol{J}_{\mathrm{M}}^{(\ell)}=\frac{\partial}{\partial z}\left[D^{(\ell)}(z, t) \frac{\partial \rho_{\mathrm{M}}^{(\ell)}}{\partial z}\right]
$$

for $0 \leq z \leq \delta(t)$ and $t \geq 0$, where $\rho_{\mathrm{M}}^{(\ell)}$ is methanol mass concentration, superscript $\ell$ represents the liquid phase, $z$ is location, $t$ is time, $\boldsymbol{J}$ is diffusive mass flux, $D^{(\ell)}$ is the binary diffusivity which depends on the molar fractions of the methanol and butanol, and $\delta(t)$ indicates the location of the moving interface or the thickness of the liquid layer. The variation of $D^{(\ell)}$ is determined by the Vignes relation [30], expressed as

$$
D^{(\ell)}=\left(D_{\mathrm{MB}}^{\mathrm{o}}\right)^{x_{\mathrm{B}}}\left(D_{\mathrm{BM}}^{\mathrm{o}}\right)^{x_{\mathrm{M}}},
$$

where the superscript ${ }^{\circ}$ represents infinitely dilute condition, and $x_{\mathrm{B}}$ and $x_{\mathrm{M}}$ are the mole fractions of 1-butanol and methanol in the liquid mixture, respectively. Both mole fractions, and thus $D^{(\ell)}$ have temporal and spatial dependency during the evaporation process. 
Figure 4 shows the extracted data of the concentration-dependent mutual diffusivity of the methanol-butanol mixture given by Shuck and Toor [31]. The solid line is from the Vignes

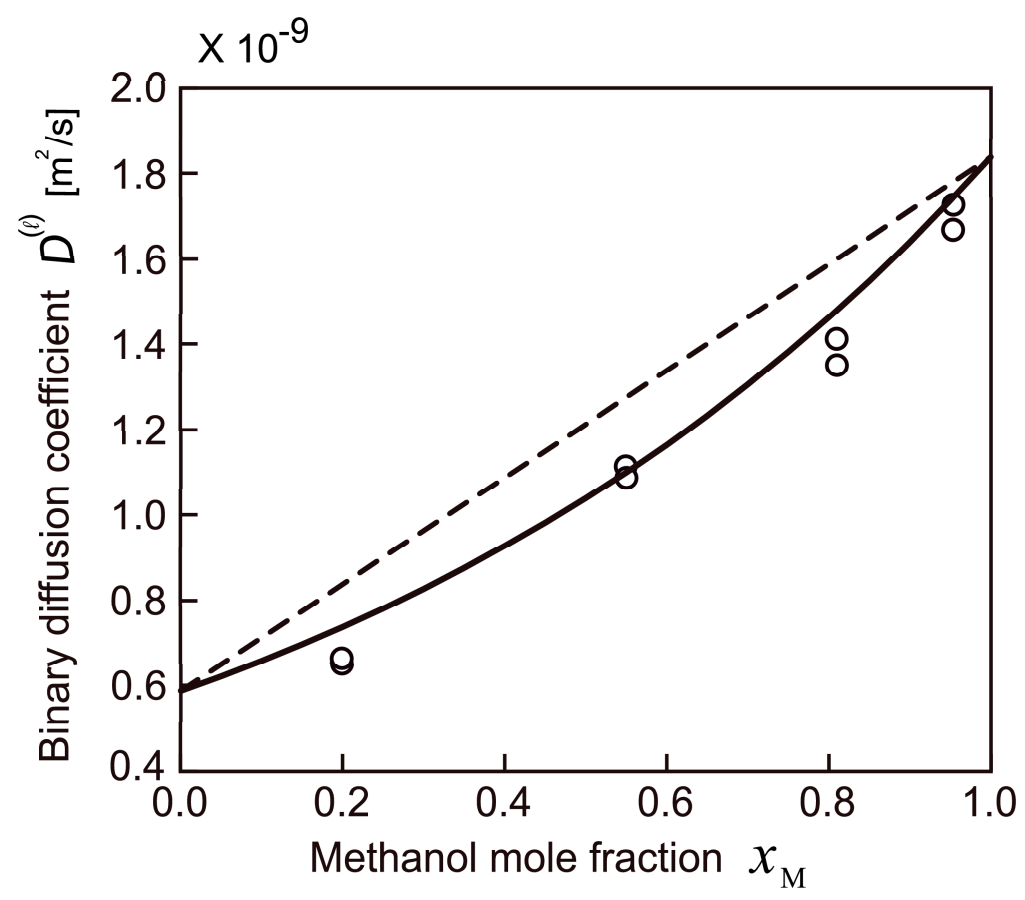

FIG. 4: Binary diffusivity in liquid phase predicted by the Vignes relation. The diffusivities of methanol and 1-butanol at infinite dilution are $D_{\mathrm{MB}}^{\mathrm{o}} \simeq 0.587 \times 10^{-9} \mathrm{~m}^{2} / \mathrm{s}$ and $D_{\mathrm{BM}}^{\circ} \simeq 1.838 \times$ $10^{-9} \mathrm{~m}^{2} / \mathrm{s}[31]$, respectively.

model while the dashed line is a reference for linear dependency. The mass concentration of methanol in the gas phase $\rho_{\mathrm{M}}^{(\mathrm{g})}$ is governed by

$$
\frac{\partial \rho_{\mathrm{M}}^{(\mathrm{g})}(z, t)}{\partial t}=-\nabla \cdot \boldsymbol{J}_{\mathrm{M}}^{(\mathrm{g})}=D^{(\mathrm{g})} \frac{\partial^{2} \rho_{\mathrm{M}}^{(\mathrm{g})}}{\partial z^{2}}-v(t) \frac{\partial \rho_{\mathrm{M}}^{(\mathrm{g})}}{\partial z}
$$

for $\delta(t) \leq z \leq b$ and $t \geq 0$, where the superscript (g) represents the gas phase, $D^{(\mathrm{g})}$ is a constant binary diffusivity, $v(t)$ is a uniform upward draft introduced by the density change across the liquid-vapor interface. The finite distance $b$ is selected in a way that $b-\delta(t)$ is approximately the long-time diffusion length. For a $1 \mathrm{D}$ system, the bulk velocity $v(t)$ is uniform everywhere in the gas domain, and it can be connected to the interface velocity based on the mass balance control volume analysis with a moving boundary, formulated as

$$
\rho^{(\ell)} \frac{\mathrm{d} \delta(t)}{\mathrm{d} t}+\rho^{(g)}\left[v(t)-\frac{\mathrm{d} \delta(t)}{\mathrm{d} t}\right]=0 \quad \text { at } \quad z=\delta,
$$


where $\rho^{(\ell)}=\rho_{\mathrm{M}}^{(\ell)}+\rho_{\mathrm{B}}^{(\ell)}$ and $\rho^{(g)}=\rho_{\mathrm{M}}^{(g)}+\rho_{\text {air }}^{(g)}$ are the total mass concentration of the liquid and gas mixtures, respectively, $d \delta / d t$ is the velocity of the moving liquid-vapor interface, and thereby

$$
v(t)=\frac{\rho^{(\mathrm{g})}-\rho^{(\ell)}}{\rho^{(\mathrm{g})}} \frac{\mathrm{d} \delta}{\mathrm{d} t} \simeq-\frac{\rho^{(\ell)}}{\rho^{(\mathrm{g})}} \frac{\mathrm{d} \delta}{\mathrm{d} t}
$$

for the vapor domain $\delta \leq z \leq b$.

The initial conditions are defined as zero methanol concentration in the gas phase, a well mixed and uniform methanol concentration in the liquid mixture, and a finite thickness $\delta_{0}$ of the liquid layer. The governing system above is subjected to four boundary conditions including two outer boundaries and two located at the liquid-vapor interface, and a compatibility integral equation to ensure the global mass conservation of the methanol. The formulations are:

(i) Zero mass flux at the crystal surface,

$$
\frac{\partial \rho_{\mathrm{M}}^{(\ell)}}{\partial z}=0 \quad \text { at } \quad z=0
$$

(ii) A presumed nearly zero methanol concentration at the outer boundary of the vapor phase domain,

$$
\rho_{\mathrm{M}}^{(\mathrm{g})} \simeq 0 \quad \text { at } \quad z=b
$$

(iii) The equilibrium partial pressure of methanol at the interface based on the Raoult's law, that is, the partial pressure is approximately the product of its mole fraction in liquid and the saturation pressure of the pure methanol, formulated as

$$
p_{\mathrm{M}}=\rho_{\mathrm{M}}^{(g)} \frac{R T}{\mathrm{M}_{\mathrm{M}}} \simeq x_{\mathrm{M}}^{(\ell)} p_{\mathrm{M}}^{\mathrm{o}}=\frac{\mathrm{M}^{(\ell)}}{\mathrm{M}_{\mathrm{M}}} \frac{\rho_{\mathrm{M}}^{(\ell)}}{\rho^{(\ell)}} p_{\mathrm{M}}^{\mathrm{o}} \quad \text { at } \quad z=\delta(t),
$$

where $p_{\mathrm{M}}$ here is the partial pressure of the methanol on the air side of the interface, $\mathrm{M}_{\mathrm{M}}$ is methanol molecular weight, $x_{\mathrm{M}}^{(\ell)}$ is methanol fraction on the liquid side of the interface, $p_{\mathrm{M}}^{\mathrm{o}}$ is the saturation pressure of pure methanol at ambient conditions, $\mathrm{M}^{(\ell)}=x_{\mathrm{M}}^{(\ell)} \mathrm{M}_{\mathrm{M}}+x_{\mathrm{B}}^{(\ell)} \mathrm{M}_{\mathrm{B}}$ is the molecular weight of the liquid mixture at the interface, $R$ is the universal gas constant, and $T$ is temperature. The above Raoult's law leads to a partition or jump condition for the methanol's mass concentration across the liquid-vapor interface:

$$
\rho_{\mathrm{M}}^{(\ell)}=\frac{\rho^{(\ell)} R T}{\mathrm{M}^{(\ell)} p_{\mathrm{M}}^{\mathrm{o}}} \rho_{\mathrm{M}}^{(\mathrm{g})} \quad \text { at } \quad z=\delta(t) .
$$


Note that the liquid mixture has an ideal behavior due to their similar molecular structure.

(iv) Mass flux balance of methanol at the moving interface,

$$
\left[\rho_{\mathrm{M}}^{(\ell)}-\rho_{\mathrm{M}}^{(\mathrm{g})}\right] \frac{\mathrm{d} \delta}{\mathrm{d} t}=-D^{(\ell)} \frac{\partial \rho_{\mathrm{M}}^{(\ell)}}{\partial z}+D^{(\mathrm{g})} \frac{\partial \rho_{\mathrm{M}}^{(\mathrm{g})}}{\partial z}-\rho_{\mathrm{M}}^{(\mathrm{g})} v
$$

at $z=\delta(t)$. The rate of change of methanol mass on the left due to evaporation is balanced by three terms on the right-hand side: the diffusive fluxes from the liquid and gas sides of the interface and the advection flux into the bulk, respectively. By replacing the bulk velocity $v$ through Eq. (5), the interfacial equation becomes

$$
\left[\rho_{\mathrm{M}}^{(\mathrm{g})} \frac{\rho^{(\ell)}}{\rho^{(\mathrm{g})}}-\rho_{\mathrm{M}}^{(\ell)}\right] \frac{\mathrm{d} \delta}{\mathrm{d} t}=D^{(\ell)} \frac{\partial \rho_{\mathrm{M}}^{(\ell)}}{\partial z}-D^{(\mathrm{g})} \frac{\partial \rho_{\mathrm{M}}^{(\mathrm{g})}}{\partial z} .
$$

(v) The compatibility condition. Solving methanol diffusion equations in both domains require knowledge of the position and velocity of the liquid-vapor interface, which, in turn, is determined by the mass concentration and fluxes in and out of the interface. A compatibility equation is developed to ensure the global mass conservation of the methanol, expressed as

$$
\begin{aligned}
& \rho^{(\ell)} \frac{\mathrm{d} \delta(t)}{\mathrm{d} t}=\frac{\mathrm{d}}{\mathrm{d} t} \int_{0}^{\delta(t)} \rho_{\mathrm{M}}^{(\ell)}(z, t) \mathrm{d} z \\
& \quad=D^{(\mathrm{g})} \frac{\partial \rho_{\mathrm{M}}^{(\mathrm{g})}(b, t)}{\partial z}-\frac{\mathrm{d}}{\mathrm{d} t} \int_{\delta(t)}^{b} \rho_{\mathrm{M}}^{(\mathrm{g})}(z, t) \mathrm{d} z,
\end{aligned}
$$

which correlates the interfacial velocity with the change of overall methanol in liquid or the change of overall methanol in gas phase plus diffusive flux at the outer boundary $(z=b)$. The overall evaporative flux $\boldsymbol{J}_{\text {Eva }}$ can be obtained from the interfacial velocity as

$$
\boldsymbol{J}_{\mathrm{Eva}}=-D^{(\ell)}(\delta, t) \frac{\partial \rho_{\mathrm{M}}^{(\ell)}(\delta, t)}{\partial z}=-\rho^{(\ell)} \frac{\mathrm{d} \delta(t)}{\mathrm{d} t}
$$

at $z=\delta$. The interfacial flux is essentially proportional to the interfacial velocity.

To facilitate the analytical approximation and numerical computation, next we consider appropriate scales and spatial coordinate transformation to immobilize the interface. By defining the spatial variable in the liquid domain as

$$
\eta=\frac{z}{\delta(t)} \quad \text { for } \quad 0 \leq z \leq \delta \quad \text { and } \quad 0 \leq \eta \leq 1 \text {, }
$$


and in vapor phase domain as

$$
\eta=1+\frac{z-\delta(t)}{b-\delta(t)} \quad \text { for } \quad \delta \leq z \leq b \quad \text { and } \quad 1 \leq \eta \leq 2
$$

for time $t \geq 0$, where $\eta$ is now a scaled spatial variable, we obtain an immobilized liquidvapor interface located at $\eta=1$. To establish an order-of-magnitude analysis, the mass concentration in liquid and gas phase can be scaled by the initial concentration of methanol in liquid phase and its corresponding vapor concentration at the interface, expressed as

$$
\rho_{\mathrm{M}}^{(\ell)} \sim \rho_{\mathrm{ini}}^{(\ell)} \quad \text { and } \quad \rho_{\mathrm{M}}^{(\mathrm{g})} \sim \frac{\mathrm{M}_{0}^{(\ell)} p_{\mathrm{M}}^{\mathrm{o}}}{\rho_{0}^{(\ell)} R T} \rho_{\mathrm{ini}}^{(\ell)},
$$

respectively, while the mass concentrations of liquid and gas mixtures can be scaled by their initial values,

$$
\rho^{(\ell)} \sim \rho_{0}^{(\ell)} \quad \text { and } \quad \rho^{(\mathrm{g})} \sim \rho_{0}^{(\mathrm{g})},
$$

respectively. The property $D^{(g)}$ is a constant, however, the methanol diffusivity in liquid phase $D^{(\ell)}$ is concentration dependent, and thus it can be scaled by the limiting value at infinite dilution,

$$
D^{(\ell)} \sim D_{\mathrm{MB}}^{\mathrm{o}} .
$$

We now consider a characteristic time $\tau$ and length $L$ for the general formulation. Both $\tau$ and $L$ later are to be selected for short- and long-time approximations. In summary, the scaled governing equations in the liquid and gas phases can be written as

$$
\frac{\partial \widehat{\rho}_{\mathrm{M}}^{(\ell)}}{\partial \widehat{t}}=-\eta\left(\frac{1}{\widehat{\delta}} \frac{\mathrm{d} \widehat{\delta}}{\mathrm{d} \widehat{t}}\right) \frac{\partial \widehat{\rho}_{\mathrm{M}}^{(\ell)}}{\partial \eta}+\frac{\tau D_{\mathrm{MB}}^{\mathrm{o}}}{L^{2}} \frac{1}{\widehat{\delta}^{2}} \frac{\partial}{\partial \eta}\left[\widehat{D}^{(\ell)} \frac{\partial \widehat{\rho}_{\mathrm{M}}^{(\ell)}}{\partial \eta}\right]
$$

for $0 \leq \eta \leq 1$, and

$$
\begin{aligned}
\frac{\partial \widehat{\rho}_{\mathrm{M}}^{(\mathrm{g})}}{\partial \widehat{t}}= & -\left(\frac{2-\eta}{\widehat{b}-\widehat{\delta}}\right) \frac{\mathrm{d} \widehat{\delta}}{\mathrm{d} \widehat{t}} \frac{\partial \widehat{\rho}_{\mathrm{M}}^{(\mathrm{g})}}{\partial \eta}+\frac{\tau D^{(\mathrm{g})}}{L^{2}}\left(\frac{1}{\widehat{b}-\widehat{\delta}}\right)^{2} \frac{\partial^{2} \widehat{\rho}_{\mathrm{M}}^{(\mathrm{g})}}{\partial \eta^{2}} \\
& -\left(\frac{1}{\widehat{b}-\widehat{\delta}}\right)\left[\frac{\widehat{\rho}^{(\mathrm{g})}-\lambda \widehat{\rho}^{(\ell)}}{\widehat{\rho}^{(\mathrm{g})}}\right] \frac{\mathrm{d} \widehat{\delta}}{\mathrm{d} \widehat{t}} \frac{\partial \widehat{\rho}_{\mathrm{M}}^{(\mathrm{g})}}{\partial \eta}
\end{aligned}
$$

for $1 \leq \eta \leq 2$, where the hat notation represents scaled variables, and $\lambda$ is the ratio of liquid and gas mixture densities defined as

$$
\lambda=\frac{\rho_{0}^{(\ell)}}{\rho_{0}^{(\mathrm{g})}} .
$$


The initial conditions are $\widehat{\rho}_{\mathrm{M}}^{(\ell)}=1$ and $\widehat{\rho}_{\mathrm{M}}^{(\mathrm{g})}=0$. The scaled boundary conditions are:

$$
\begin{gathered}
\frac{\partial \widehat{\rho}_{\mathrm{M}}^{(\ell)}}{\partial \eta}=0 \quad \text { at } \quad \eta=0, \\
\widehat{\rho}_{\mathrm{M}}^{(\mathrm{g})}=0 \quad \text { at } \quad \eta=2, \\
\widehat{\rho}_{\mathrm{M}}^{(\ell)}=\frac{\widehat{\rho}^{(\ell)}}{\widehat{\mathrm{M}}^{(\ell)}} \widehat{\rho}_{\mathrm{M}}^{(\mathrm{g})} \quad \text { at } \quad \eta=1,
\end{gathered}
$$

where $\widehat{\mathrm{M}}^{(\ell)}=\mathrm{M}^{(\ell)} / \mathrm{M}_{0}^{(\ell)}$, and

$$
\begin{aligned}
& {\left[\lambda \gamma \frac{\widehat{\rho}^{(\ell)}}{\hat{\rho}^{(\mathrm{g})}} \widehat{\rho}_{\mathrm{M}}^{(\mathrm{g})}-\widehat{\rho}_{\mathrm{M}}^{(\ell)}\right] \frac{\mathrm{d} \widehat{\delta}}{\mathrm{d} \widehat{t}}} \\
& \quad=\frac{\tau D_{\mathrm{MB}}^{\mathrm{o}}}{L^{2}} \frac{\widehat{D}^{(\ell)}}{\widehat{\delta}} \frac{\partial \widehat{\rho}_{\mathrm{M}}^{(\ell)}}{\partial \eta}-\frac{\tau D^{(\mathrm{g})}}{L^{2}}\left(\frac{\gamma}{\widehat{b}-\widehat{\delta}}\right) \frac{\partial \widehat{\rho}_{\mathrm{M}}^{(\mathrm{g})}}{\partial \eta}
\end{aligned}
$$

at $\eta=1$. The essential dimensionless group $\gamma$ that controls the partition condition through the saturation vapor pressure at the interface is defined as

$$
\gamma=\frac{\mathrm{M}_{0}^{(\ell)} p_{\mathrm{M}}^{o}}{\rho_{0}^{(\ell)} R T} .
$$

Furthermore, the compatibility condition, Eq. (12), can be written as

$$
\begin{aligned}
& \hat{\rho}^{(\ell)} \frac{\mathrm{d} \widehat{\delta}}{\mathrm{d} \widehat{t}}=\omega_{\mathrm{M}}^{0} \frac{\mathrm{d}}{\mathrm{d} \widehat{t}} \int_{0}^{\widehat{\delta}} \widehat{\rho}_{\mathrm{M}}^{(\ell)}(\eta, \widehat{t}) \mathrm{d} \eta \\
& =\frac{\tau D^{(\mathrm{g})}}{L^{2}}\left(\frac{\omega_{\mathrm{M}}^{\mathrm{o}} \gamma}{\widehat{b}-\widehat{\delta}}\right) \frac{\partial \widehat{\rho}_{\mathrm{M}}^{(\mathrm{g})}(2, \widehat{t})}{\partial \eta}-\omega_{\mathrm{M}}^{\mathrm{o}} \gamma \frac{\mathrm{d}}{\mathrm{d} \widehat{t}} \int_{\widehat{\delta}}^{\widehat{b}} \widehat{\rho}_{\mathrm{M}}^{(\mathrm{g})}(\eta, \widehat{t}) \mathrm{d} \eta,
\end{aligned}
$$

where $\omega_{\mathrm{M}}^{\mathrm{o}}=\rho_{\mathrm{ini}}^{(\ell)} / \rho_{0}^{(\ell)}$ is initial mass fraction of methanol. Next we present the analytical approximations of the nonlinear system in the short- and long-time regimes.

\section{B. Short-time Approximation}

The length and time scales for the short-time approximation are defined as

$$
L=b-\delta_{0} \quad \text { and } \quad \tau=\frac{\left(b-\delta_{0}\right)^{2}}{D^{(\mathrm{g})}},
$$

respectively, which correspond to diffusion in the gas phase. Therefore Eqs. (19) and (20) can be written as

$$
\frac{\partial \widehat{\rho}_{\mathrm{M}}^{(\ell)}}{\partial \widehat{t}}=-\eta\left(\frac{1}{\widehat{\delta}} \frac{\mathrm{d} \widehat{\delta}}{\mathrm{d} \widehat{t}}\right) \frac{\partial \widehat{\rho}_{\mathrm{M}}^{(\ell)}}{\partial \eta}+\frac{\alpha}{\widehat{\delta}^{2}} \frac{\partial}{\partial \eta}\left[\widehat{D}^{(\ell)} \frac{\partial \widehat{\rho}_{\mathrm{M}}^{(\ell)}}{\partial \eta}\right],
$$


for $0 \leq \eta \leq 1$, and

$$
\begin{aligned}
\frac{\partial \widehat{\rho}_{\mathrm{M}}^{(\mathrm{g})}}{\partial \widehat{t}}= & -\left(\frac{2-\eta}{\widehat{b}-\widehat{\delta}}\right) \frac{\mathrm{d} \widehat{\delta}}{\mathrm{d} \widehat{t}} \frac{\partial \widehat{\rho}_{\mathrm{M}}^{(\mathrm{g})}}{\partial \eta}+\left(\frac{1}{\widehat{b}-\widehat{\delta}}\right)^{2} \frac{\partial^{2} \widehat{\rho}_{\mathrm{M}}^{(\mathrm{g})}}{\partial \eta^{2}} \\
& -\left(\frac{1}{\widehat{b}-\widehat{\delta}}\right)\left[\frac{\hat{\rho}^{(\mathrm{g})}-\lambda \widehat{\rho}^{(\ell)}}{\widehat{\rho}^{(\mathrm{g})}}\right] \frac{\mathrm{d} \widehat{\delta}}{\mathrm{d} \widehat{t}} \frac{\partial \widehat{\rho}_{\mathrm{M}}^{(\mathrm{g})}}{\partial \eta}
\end{aligned}
$$

for $1 \leq \eta \leq 2, \widehat{t} \geq 0$. Where $\alpha$ is the ratio of diffusivities in liquid and gas phases,

$$
\alpha=\frac{D_{\mathrm{MB}}^{\mathrm{o}}}{D^{(\mathrm{g})}} .
$$

In the short-time limit, the very small change of concentration in the liquid phase has been neglected in the approximation, and thus the selection of compatibility condition in Eq. (27) is based on the integral in the gas phase domain, expressed as

$$
\begin{aligned}
\widehat{\rho}^{(\ell)} \frac{\mathrm{d} \widehat{\delta}}{\mathrm{d} \widehat{t}}= & \left(\frac{\omega_{\mathrm{M}}^{\mathrm{o}} \gamma}{\widehat{b}-\widehat{\delta}}\right) \frac{\partial \widehat{\rho}_{\mathrm{M}}^{(\mathrm{g})}(2, \widehat{t})}{\partial \eta} \\
& -\omega_{\mathrm{M}}^{\mathrm{o}} \gamma \frac{\mathrm{d}}{\mathrm{d} \widehat{t}} \int_{\widehat{\delta}}^{\widehat{b}} \widehat{\rho}_{\mathrm{M}}^{(\mathrm{g})}(\eta, \widehat{t}) \mathrm{d} \eta .
\end{aligned}
$$

Now considering a test case with 50/50 wt $\%$ mixture and basic constants given in Table I, we obtain $\alpha \simeq 3.89 \times 10^{-5}, \widehat{b}=2, \lambda \simeq 645.16, \gamma \simeq 3.83 \times 10^{-4}$, also $\omega_{\mathrm{M}}^{\mathrm{o}}=0.5, \mathrm{M}^{(\ell)} \rightarrow \mathrm{M}_{0}^{(\ell)}$, $\rho^{(\ell)} \rightarrow \rho_{0}^{(\ell)}, \rho^{(\mathrm{g})} \rightarrow \rho_{0}^{(\mathrm{g})}$, and the change of methanol concentration in liquid phase $\Delta \hat{\rho}_{\mathrm{M}}^{(\ell)}$ is about $\mathcal{O}\left(10^{-2}\right)$, while in the gas phase $\Delta \widehat{\rho}_{\mathrm{M}}^{(\mathrm{g})}$ is around $\mathcal{O}(1)$. By comparing the order of magnitudes in Eqs. (29), (30), and (32), the leading-order approximations of the diffusion equations become

$$
\frac{\partial \widehat{\rho}_{M}^{(\ell)}}{\partial \widehat{t}} \simeq 0 \text { for } 0 \leq \eta \leq 1
$$

and

$$
\frac{\partial \widehat{\rho}_{\mathrm{M}}^{(\mathrm{g})}}{\partial \widehat{t}} \simeq\left(\frac{1}{\widehat{b}-\widehat{\delta}}\right)^{2} \frac{\partial^{2} \widehat{\rho}_{\mathrm{M}}^{(\mathrm{g})}}{\partial \eta^{2}} \text { for } 1 \leq \eta \leq 2 .
$$

For uniformly distributed initial concentration in the liquid phase, the solution of Eq. (33) is $\hat{\rho}_{\mathrm{M}}^{(\ell)}=1$. Applying Laplace transformation to Eq. (34), the governing equation in the Laplace domain becomes

$$
\bar{\rho}^{\prime \prime}(s, \eta)-(\widehat{b}-\widehat{\delta})^{2} s \bar{\rho}(s, \eta)=0,
$$

where $\bar{\rho}$ is the transformed $\hat{\rho}_{\mathrm{M}}^{(\mathrm{g})}, s$ is the frequency variable, and the double prime is the second order derivatives with respect to $\eta$. Considering the far-field boundary condition Eq. 


\begin{tabular}{lcr}
\hline Parameter & Symbol & Value, SI \\
\hline Mass conc. of pure methanol [32] & $\rho_{\mathrm{M}}^{\mathrm{o}}$ & $787.2 \mathrm{~kg} / \mathrm{m}^{3}$ \\
Mass conc. of pure 1-butanol [33] & $\rho_{\mathrm{B}}^{\mathrm{o}}$ & $805.9 \mathrm{~kg} / \mathrm{m}^{3}$ \\
Initial conc. of gas mixture (air) & $\rho_{0}^{(\mathrm{g})}$ & $1.24 \mathrm{~kg} / \mathrm{m}^{3}$ \\
Initial conc. of liquid mixture & $\rho_{0}^{(\ell)}$ & $797 \mathrm{~kg} / \mathrm{m}^{3}$ \\
Initial conc. of methanol & $\rho_{\mathrm{ini}}^{(\ell)}$ & $400 \mathrm{~kg} / \mathrm{m}^{3}$ \\
Molar mass of methanol [32] & $\mathrm{M}_{\mathrm{M}}$ & $32.04 \mathrm{~kg} / \mathrm{kmol}$ \\
Molar mass of 1-butanol [33] & $\mathrm{M}_{\mathrm{B}}$ & $74.12 \mathrm{~kg} / \mathrm{kmol}$ \\
Initial liquid molar mass & $\mathrm{M}_{0}^{(\ell)}$ & $44.74 \mathrm{~kg} / \mathrm{kmol}$ \\
Sat. pressure of methanol [28] & $p_{\mathrm{M}}^{o}$ & $16981 \mathrm{~Pa}$ \\
Viscosity of pure methanol [32] & $\eta_{\mathrm{M}}$ & $5.45 \times 10^{-4} \mathrm{~Pa} \mathrm{~s}$ \\
Viscosity of pure 1-butanol [33] & $\eta_{\mathrm{B}}$ & $2.573 \times 10^{-3} \mathrm{~Pa} \mathrm{~s}$ \\
Diffusivity in gas & $D^{(\mathrm{g})}$ & $1.51 \times 10^{-5} \mathrm{~m}{ }^{2} / \mathrm{s}$ \\
Universal gas constant & $R$ & $8.314 \mathrm{~J} / \mathrm{mol}-\mathrm{K}$ \\
Temperature & $T$ & $298.15 \mathrm{~K}$ \\
\hline
\end{tabular}

TABLE I: Basic constants and parameters used in the scaling analysis.

(23) and the partition condition Eq. (24), the analytical solution of Eq. (35) is

$$
\bar{\rho}(\eta)=\frac{\widehat{\mathrm{M}}^{(\ell)}}{\widehat{\rho}^{(\ell)}} \frac{\phi(s, \eta-1)}{s[1-\phi(s, 2)]}-\frac{\widehat{\mathrm{M}}^{(\ell)}}{\widehat{\rho}^{(\ell)}} \frac{\phi(s, 3-\eta)}{s[1-\phi(s, 2)]},
$$

where

$$
\phi(s, \eta)=\exp [(\widehat{\delta}-\widehat{b}) \eta \sqrt{s}]<1 .
$$

Thus by expanding $[1-\phi(s, 2)]^{-1}$ to an series form,

$$
[1-\phi(s, 2)]^{-1}=\sum_{n=0}^{\infty} \phi(s, 2 n),
$$

Eq. (36) can be expressed as

$$
\begin{aligned}
\bar{\rho}(s, \eta)= & \frac{\widehat{\mathrm{M}}^{(\ell)}}{s \widehat{\rho}^{(\ell)}} \sum_{n=0}^{\infty} \phi(s, 2 n+\eta-1) \\
& -\frac{\widehat{\mathrm{M}}^{(\ell)}}{s \widehat{\rho}^{(\ell)}} \sum_{n=0}^{\infty} \phi(s, 2 n-\eta+3) .
\end{aligned}
$$


By taking the inverse Laplace transform, we obtain the analytical solution:

$$
\begin{aligned}
\widehat{\rho}_{\mathrm{M}}^{(\mathrm{g})}(\eta, \widehat{t})= & \frac{\widehat{\mathrm{M}}^{(\ell)}}{\widehat{\rho}^{(\ell)}} \sum_{n=0}^{\infty} \operatorname{erfc}\left[\frac{(\widehat{b}-\widehat{\delta})(2 n+\eta-1)}{2 \sqrt{\hat{t}}}\right] \\
& -\frac{\widehat{\mathrm{M}}^{(\ell)}}{\widehat{\rho}^{(\ell)}} \sum_{n=0}^{\infty} \operatorname{erfc}\left[\frac{(\widehat{b}-\widehat{\delta})(2 n-\eta+3)}{2 \sqrt{\hat{t}}}\right]
\end{aligned}
$$

for $1 \leq \eta \leq 2$ and $\widehat{t}>0$. As a result, the interface position can also be estimated by the compatibility condition, Eq. (32). The short-time solution provides an analytical approximation of the transient evaporation kinetics at the very beginning stage.

\section{Long-time Approximation}

The length and time scales for the long-time approximation are defined as

$$
L=\delta_{0} \quad \text { and } \quad \tau=\frac{\delta_{0}^{2}}{D_{\mathrm{MB}}^{\circ}},
$$

respectively, which corresponds to diffusion in the liquid phase. Thus Eqs. (19) and (20) become

$$
\frac{\partial \widehat{\rho}_{\mathrm{M}}^{(\ell)}}{\partial \widehat{t}}=-\eta\left(\frac{1}{\widehat{\delta}} \frac{\mathrm{d} \widehat{\delta}}{\mathrm{d} \widehat{t}}\right) \frac{\partial \widehat{\rho}_{\mathrm{M}}^{(\ell)}}{\partial \eta}+\frac{1}{\widehat{\delta}^{2}} \frac{\partial}{\partial \eta}\left[\widehat{D}^{(\ell)} \frac{\partial \widehat{\rho}_{\mathrm{M}}^{(\ell)}}{\partial \eta}\right]
$$

for $0 \leq \eta \leq 1$, and

$$
\begin{aligned}
\frac{\partial \widehat{\rho}_{\mathrm{M}}^{(\mathrm{g})}}{\partial \widehat{t}}= & -\left(\frac{2-\eta}{\widehat{b}-\widehat{\delta}}\right) \frac{\mathrm{d} \widehat{\delta}}{\mathrm{d} \widehat{t}} \frac{\partial \widehat{\rho}_{\mathrm{M}}^{(\mathrm{g})}}{\partial \eta}+\frac{1}{\alpha}\left(\frac{1}{\widehat{b}-\widehat{\delta}}\right)^{2} \frac{\partial^{2} \widehat{\rho}_{\mathrm{M}}^{(\mathrm{g})}}{\partial \eta^{2}} \\
& -\left(\frac{1}{\widehat{b}-\widehat{\delta}}\right)\left[\frac{\widehat{\rho}^{(\mathrm{g})}-\lambda \widehat{\rho}^{(\ell)}}{\widehat{\rho}^{(\mathrm{g})}}\right] \frac{\mathrm{d} \widehat{\delta}}{\mathrm{d} \widehat{t}} \frac{\partial \widehat{\rho}_{\mathrm{M}}^{(\mathrm{g})}}{\partial \eta}
\end{aligned}
$$

for $1 \leq \eta \leq 2, \widehat{t} \geq 0$. Also the interfacial flux balance condition is

$$
\left[\lambda \gamma \frac{\widehat{\rho}^{(\ell)}}{\widehat{\rho}^{(\mathrm{g})}} \widehat{\rho}_{\mathrm{M}}^{(\mathrm{g})}-\widehat{\rho}_{\mathrm{M}}^{(\ell)}\right] \frac{\mathrm{d} \widehat{\delta}}{\mathrm{d} \widehat{t}}=\frac{\widehat{D}^{(\ell)}}{\widehat{\delta}} \frac{\partial \widehat{\rho}_{\mathrm{M}}^{(\ell)}}{\partial \eta}-\frac{1}{\alpha}\left(\frac{\gamma}{\widehat{b}-\widehat{\delta}}\right) \frac{\partial \widehat{\rho}_{\mathrm{M}}^{(\mathrm{g})}}{\partial \eta}
$$

at $\eta=1$. Similarly by carrying out order of magnitude analysis for the long-time model above, the leading-order approximation leads to the simplified governing equations:

$$
\frac{\partial \widehat{\rho}_{\mathrm{M}}^{(\ell)}}{\partial \widehat{t}} \simeq \frac{\widehat{D}^{(\ell)}}{\widehat{\delta}^{2}} \frac{\partial^{2} \widehat{\rho}_{\mathrm{M}}^{(\ell)}}{\partial \eta^{2}} \quad \text { for } \quad 0 \leq \eta \leq 1
$$

and

$$
\frac{\partial^{2} \widehat{\rho}_{\mathrm{M}}^{(\mathrm{g})}}{\partial \eta^{2}} \simeq 0 \quad \text { for } \quad 1 \leq \eta \leq 2
$$


Because of the small interface velocity, the mass flux balance Eq. (44) can be approximated as

$$
\frac{\partial \widehat{\rho}_{\mathrm{M}}^{(\ell)}}{\partial \eta} \simeq \frac{\widehat{\delta}}{\alpha}\left(\frac{\gamma}{\widehat{b}-\widehat{\delta}}\right) \frac{\partial \widehat{\rho}_{\mathrm{M}}^{(\mathrm{g})}}{\partial \eta} .
$$

Considering boundary conditions (22), (24), and (47), we obtain the following analytical solution:

$$
\begin{aligned}
& \widehat{\rho}_{\mathrm{M}}^{(\ell)}(\eta, \widehat{t})= \sum_{\mathrm{n}=1}^{\infty} C_{\mathrm{n}} \frac{\sqrt{\widehat{D}^{(\ell)}} \cos \left(\widehat{\delta} \eta \beta_{\mathrm{n}} / \sqrt{\widehat{D}^{(\ell)}}\right) \gamma}{\alpha(\widehat{b}-\widehat{\delta}) \beta_{n} \sin \left(\widehat{\delta} \beta_{n} / \sqrt{\widehat{D}^{(\ell)}}\right)} \mathrm{e}^{-\beta_{\mathrm{n}}^{2} \hat{t}}, \\
& \hat{\rho}_{\mathrm{M}}^{(\mathrm{g})}(\eta, \widehat{t})=\sum_{\mathrm{n}=1}^{\infty} C_{\mathrm{n}}(2-\eta) \mathrm{e}^{-\beta_{\mathrm{n}}^{2} \hat{t}},
\end{aligned}
$$

where the eigenvalues $\beta_{\mathrm{n}}$ can be computed from the transcendental equation:

$$
\tan \left[\frac{\widehat{\delta} \beta_{n}}{\sqrt{\widehat{D}^{(\ell)}}}\right]=\frac{\gamma \widehat{\mathrm{M}}^{(\ell)} \sqrt{\widehat{D}^{(\ell)}}}{\widehat{\rho}^{(\ell)} \alpha(\widehat{b}-\widehat{\delta}) \beta_{n}},
$$

in which the first five eigenvalues applied in the computation are $\beta_{1}=2.6619, \beta_{2}=8.1404$, $\beta_{3}=13.8953, \beta_{4}=19.8525$, and $\beta_{5}=25.9276$. The dimensionless interface position can also be tracked by the compatibility condition (27) and to obtain the evaporation flux.

\section{EXPERIMENT}

The evaporation kinetics and the change of local viscosity of the liquid mixture are measured by the impedance response of the combined shear and longitudinal waves. Here we briefly summarize the experimental procedures, conditions, measurement methods, and instrumentation principles.

Crystal resonator and sample preparation. The crystal resonators were purchased from Stanford Research Systems (SRS). These were polished AT-cut quartz crystals with gold electrodes on each surface and a fundamental resonant frequency about $5 \mathrm{MHz}$. The crystal diameter was around $2.4 \mathrm{~cm}$, thickness around $0.5 \mathrm{~mm}$, the top and bottom electrode diameters were 1.2 and $0.6 \mathrm{~cm}$, respectively. The coated electrodes were to provide alternating current and voltage to the piezoelectric quartz crystal and introduce simultaneous shear and longitudinal oscillations. The crystal was placed in a teflon holder for submerging the crystal in liquids without contact to the lower electrode. The crystal holder was connected to the SRS QCM25 oscillator through a coaxial joint and a CAT5 cable connected 
the oscillator to the SRS QCM200 controller with a built-in frequency counter and resistance meter. The controller was connected to a computer through RS-232 for data acquisition. A LabView (National Instruments) module provided by SRS was used to record the resonant frequency and resistance of the crystal at a sampling rate of $1 \mathrm{~s}^{-1}$. Reagent-grade methanol and 1-butanol purchased from Sigma Aldrich were used as provided without any extra purifications steps taken. The mixtures were prepared on a basis of mass fraction and were mixed using a vortex mixer at low speed. The sample were then used immediately to minimize composition changes due to evaporation. Each experiment was performed at ambient conditions and the temperature was $25 \pm 2^{\circ} \mathrm{C}$. Before each experiment, the bare crystal was placed in the holder and if the unloaded resonant frequency varied by $\pm 50 \mathrm{~Hz}$ from it's original resonant frequency, the crystal was cleaned with de-ionized water and acetone, and then checked again. If the resonant frequency still differed, the crystal was discarded. For each experiment, the data logging was started immediately after $1 \mathrm{~mL}$ of the sample was pipetted onto the surface of the crystal. After each trial, the crystal was rinsed in acetone to remove any residues followed by de-ionized water and dried with nitrogen gas. Extra care had been taken not to touch the electrodes while holding the crystal with nitrile groves to avoid any abrasions on the surface of the electrode which may have a noticeable effect on the impedance or frequency response.

Longitudinal mode and spikes on the resonant frequency shift. The appearance of frequency spikes including its amplitude and spike shape are not yet fully understood. The amplitude of the spikes varies but the wave form is quite similar during evaporation. Physically the acoustic wave is due to unavoidable longitudinal crystal oscillation triggered along with the shear motion of the resonator, and will resonate with the free surface [34]. Specifically the longitudinal wave emits from the crystal surface, travels upward, and then reflects at the free surface. The reflective wave interacts with the emitting wave constructively and destructively, resulting in resonant spikes. Each spike appears at an incremental film thickness that is multiples of the half wave length of the acoustic wave in liquid [79, 17, 35]. The longitudinal wave creates the spikes on the resonant frequency shift (Fig. 2). Depending on the liquid density and compressibility, the acoustic speed is on the order of $10^{3} \mathrm{~m} / \mathrm{s}$. The reduction of the thickness corresponds to two consecutive spikes is half of the acoustic wave length, around tens of microns. Therefore, the interfacial moving velocity can 
be estimated through the time difference $\triangle t$ between two consecutive spikes, formulated as

$$
\frac{\mathrm{d} \delta}{\mathrm{d} t} \simeq \frac{-\lambda_{\mathrm{a}}}{2 \triangle t} \simeq \frac{-\pi}{\left(f_{0}+\triangle f\right) \triangle t}\left[\rho^{(\ell)} \mathcal{K}\right]^{-1 / 2},
$$

where $\mathcal{K}$ is isentropic compressibility of the liquid mixture and $\lambda_{\mathrm{a}}$ is the acoustic wave length. The isentropic compressibility of pure methanol and 1-butanol at room temperature are $\mathcal{K}_{\mathrm{M}}=10.446 \times 10^{-10} \mathrm{~Pa}^{-1}[36]$ and $\mathcal{K}_{\mathrm{B}}=8.082 \times 10^{-10} \mathrm{~Pa}^{-1}[37]$, respectively. The isentropic compressibility of the mixture can be calculated by a simple linear combination [36, 37],

$$
\mathcal{K}=x_{\mathrm{M}} \mathcal{K}_{\mathrm{M}}+x_{\mathrm{B}} \mathcal{K}_{\mathrm{B}},
$$

where $x_{\mathrm{M}}$ and $x_{\mathrm{M}}$ are the average molar fractions of methanol and 1-butanol respectively. The amplitude and wave form of each spike are not considered here.

Shear mode and resonant frequency shift. The shear motion of the crystal resonator is driven at its resonant frequency, which is determined by the thickness and material properties of the the crystal disc. The shear flow is driven by a periodic oscillatory motion of the crystal. The liquid mixture deposited on the crystal surface thus leads to a decrease of resonant frequency. The amount of the resonant frequency shift connects the liquid property such as density and viscosity to the mechanical impedance on the crystal surface, that is, hydrodynamic drag acting on the crystal surface during the shear oscillation. The penetration depth of the shear wave is only a few hundreds of nanometers at megahertz frequency, and thus the shear wave is in a semi-infinite domain. This is a typical Stokes 2nd problem. The momentum equation reduces to a linear diffusive equation, describing the diffusive transport of vorticity into the fluid due to the viscous effect. Therefore by coupling the equation of motion of both fluid and crystal, Kanazawa and Gordon established the relationship between the resonant frequency shift, material properties of the crystal resonator, and the liquid properties of a Newtonian sample fluid [15]. Based on the small-load approximation the new resonant condition leads to the following result on the shift of resonant frequency:

$$
\triangle f \simeq-f_{0}^{3 / 2}\left(\frac{\eta_{\mathrm{L}} \rho^{(\ell)}}{\pi \mu_{\mathrm{Q}} \rho_{\mathrm{Q}}}\right)^{1 / 2},
$$

where $\triangle f$ is the negative frequency shift of loaded crystal, and $f_{0}$ is the resonant frequency of a bare crystal, $\eta_{\mathrm{L}}$ (defined in Eq. 54) is the viscosity and $\rho^{(\ell)}$ is the total mass concentration of the liquid near the crystal surface, $\mu_{\mathrm{Q}} \simeq 2.947 \times 10^{10} \mathrm{~kg} \mathrm{~m}^{-1} \mathrm{~s}^{-2}$ is the shear modulus and $\rho_{\mathrm{Q}} \simeq 2648 \mathrm{~kg} / \mathrm{m}^{3}$ is the mass density of the crystal, and the small-load approximation 
is applicable when $|\triangle f| \ll f_{0}$. The relationship manifests that the frequency shift is proportional to the square root of the liquid viscosity and density. The shift of the resonant frequency therefore indicates the change of the local viscosity and density of the mixture near the crystal surface. The characteristic viscous penetration depth, a few hundred nanometers for a resonant frequency of $5 \mathrm{MHz}$, is much less than the thickness of the whole liquid layer. Therefore the properties measured are approximately located at $z \simeq 0$. The amplitude of the displacement at the center of the crystal surface is about 1 to $2 \mathrm{~nm}$, which corresponds to a velocity amplitude around 3 to $5 \mathrm{~cm} / \mathrm{s}$, and thus the Reynolds number is $\mathcal{O}\left(10^{-5}\right)$.

\section{RESULTS AND DISCUSSION}

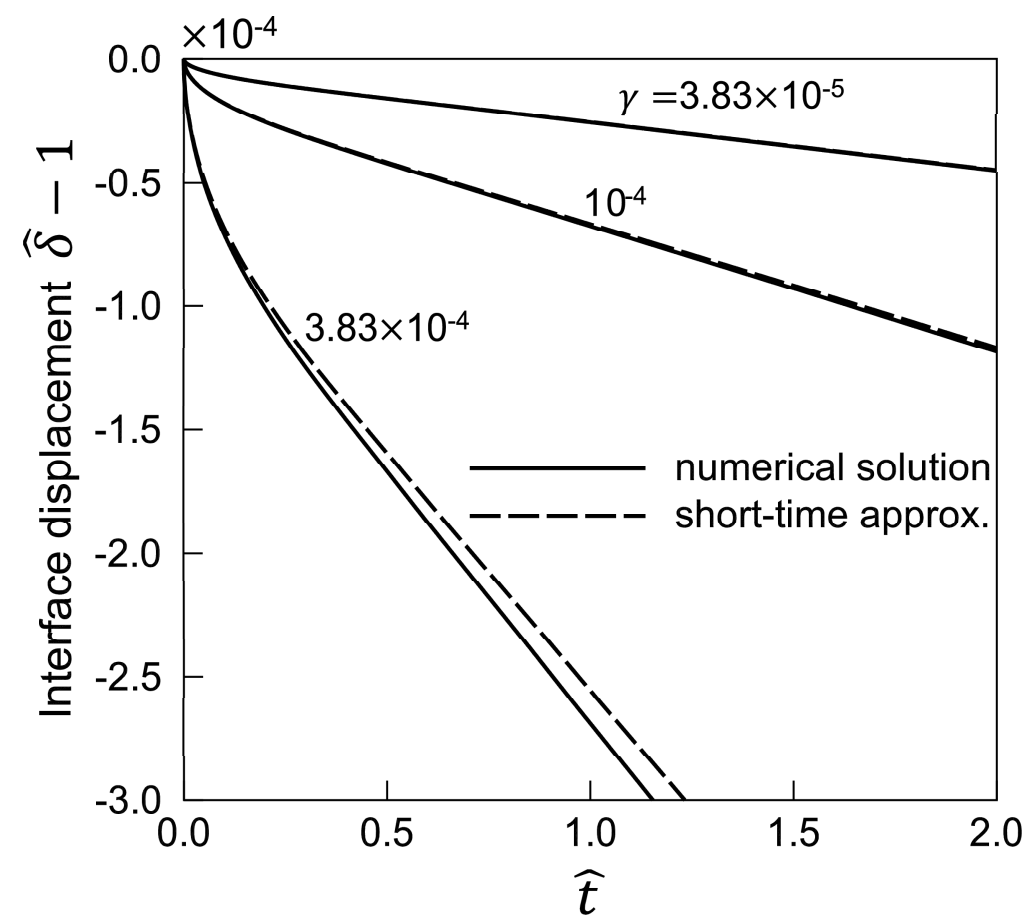

FIG. 5: Reduction of the thickness of the liquid layer or the displacement of the liquid-vapor interface versus time at the initial stage of evaporation. Parameters used are: $\alpha=3.89 \times 10^{-5}$, $\gamma=3.83 \times 10^{-4}, 1.0 \times 10^{-4}$, and $3.83 \times 10^{-5}, \lambda=645.16, \widehat{b}=2.0$, and $\omega_{\mathrm{M}}^{\mathrm{o}}=0.5$. In the short-time limit the time $\widehat{t}$ is scaled by $\left(b-\delta_{0}\right)^{2} / D^{(\mathrm{g})}$, and thickness $\widehat{\delta}$ is scaled by $b-\delta_{0}$.

Figure 5 shows the transient displacement of the liquid-vapor interface near the initial stage of evaporation. The short-time approximation provides leading order asymptotic curves (dashed lines) for the computational results (solid lines) based on the nonlinear model. 


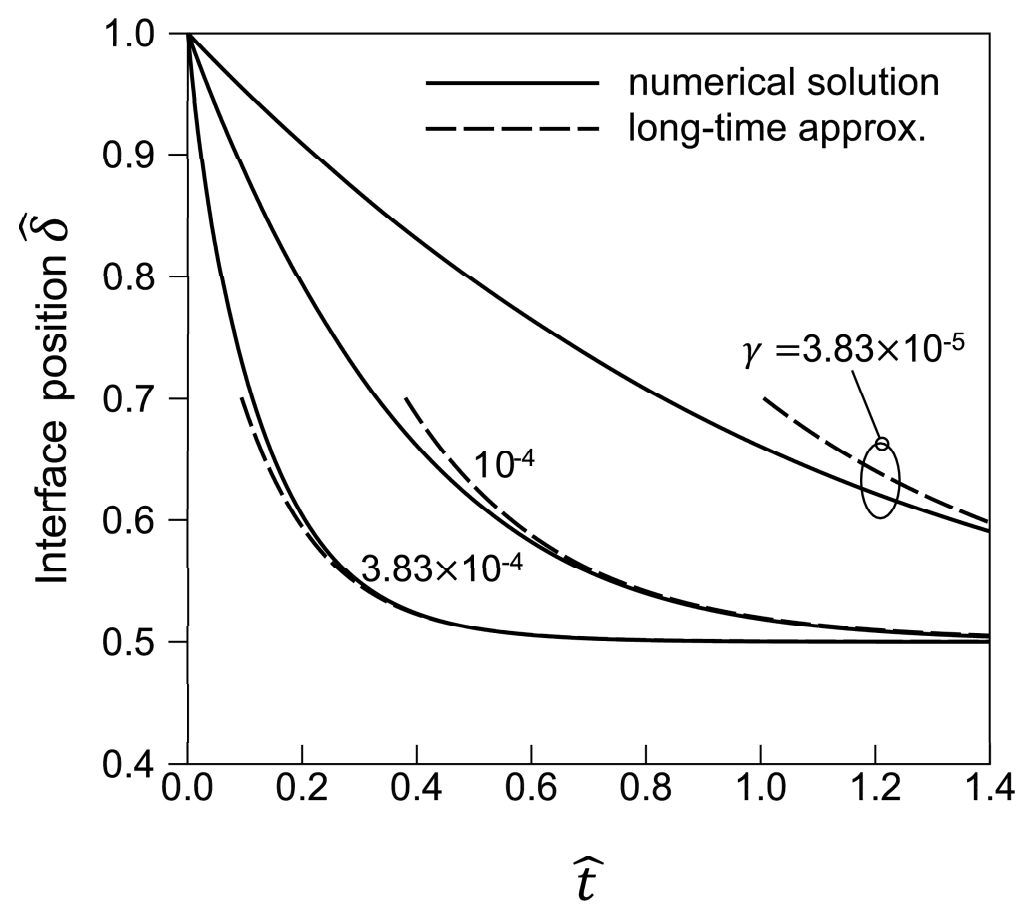

FIG. 6: The displacement of the liquid-vapor interface versus time. Parameters used are the same as in Fig. 5 . In the long-time limit, the time $\widehat{t}$ is scaled by $\delta_{0}^{2} / D_{\mathrm{MB}}^{\mathrm{o}}$, and thickness $\widehat{\delta}$ is scaled by $\delta_{0}$.

The numerical result is carried out by the fully implicit Crank-Nicolson scheme [38] along with LU decomposition for the matrix solver [39]. The analytical approximation slightly underpredicts the evaporation rate or the interfacial moving speed primarily due to the neglected bulk velocity that assists the evaporation. In the short-time limit evaporation is mainly controlled by the mass transport in the gas phase, while the assumed constant variables, $D^{(\ell)}, \mathrm{M}^{(\ell)}, \rho^{(\ell)}$ and $\rho^{(\mathrm{g})}$ have relatively less influence on the evaporation rate. A relatively large concentration gradient appears near the initial point. This is due to a step function assigned to the initial concentration profile, which leads to a fast evaporation rate. The moving interface slows down and the evaporation rate reaches a quasi-linear behavior afterward. The test case based on Table I has $\gamma=3.83 \times 10^{-4}$, another two $\gamma$ values corresponding to, for example different saturation vapor pressure, are references for the sensitivity test of the partition condition. It is found that the deviation between the analytical approximation and numerical result decreases as the partition number $\gamma$ decreases, meaning the gas-phase dominated leading-order approximation is more accurate for cases with lower saturation pressure at the interface. This is due to a smaller error introduced by the neglected bulk velocity corresponding to a smaller evaporation flux. 

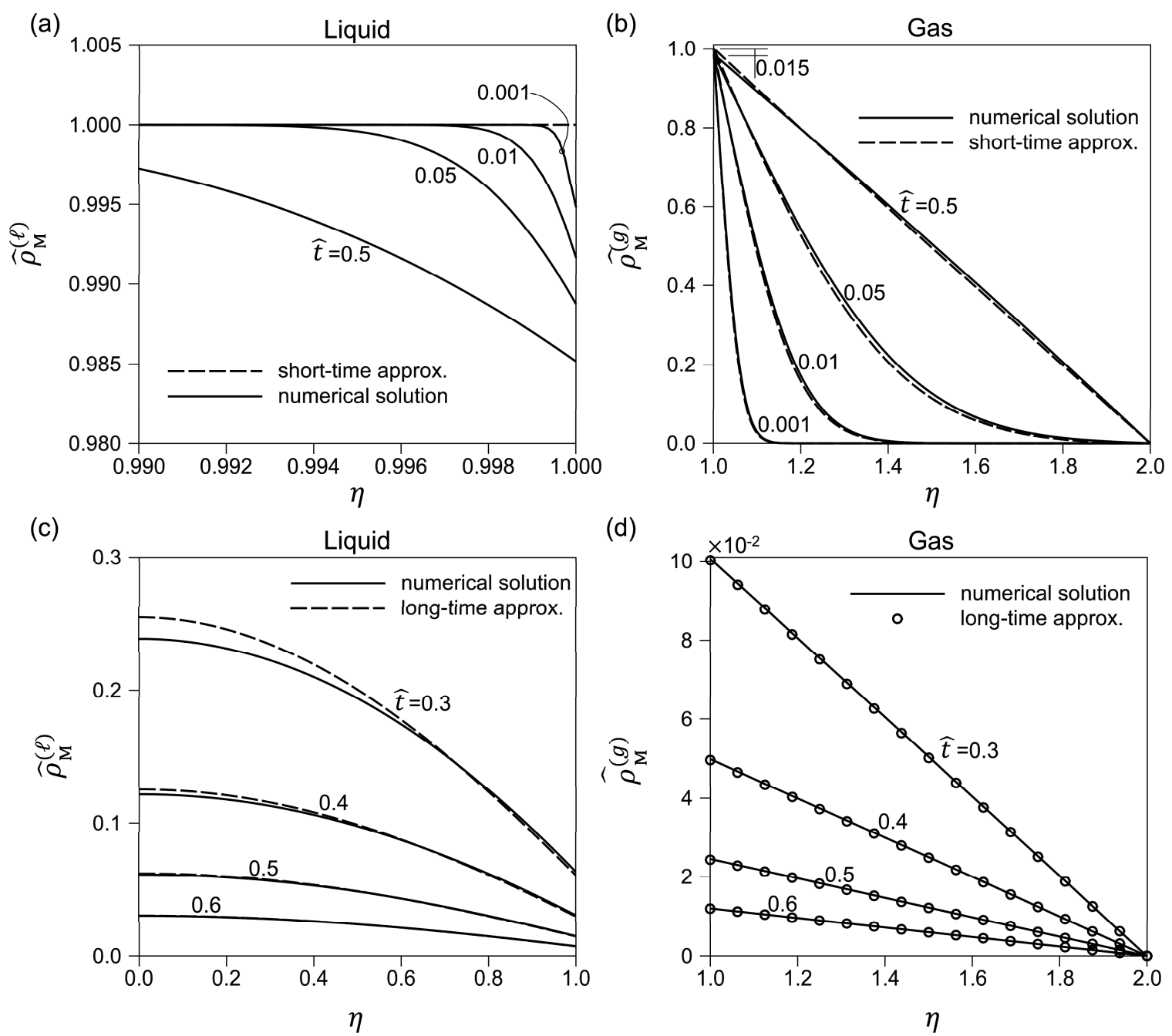

FIG. 7: Transient profiles of methanol mass concentration in both liquid and gas phase domains in the short- $(\mathrm{a}, \mathrm{b})$ and long-time $(\mathrm{c}, \mathrm{d})$ regimes. The leading-order analytical approximations are compared with the full numerical solution. Parameters used in this case are: $\alpha=3.89 \times 10^{-5}$, $\gamma=3.83 \times 10^{-4}, \lambda=645.16, \widehat{b}=2.0$, and $\omega_{\mathrm{M}}^{\mathrm{o}}=0.5$. In (a) and (b), the time $\widehat{t}$ is scaled by $\left(b-\delta_{0}\right)^{2} / D^{(\mathrm{g})}$, and thickness $\widehat{\delta}$ is scaled by $b-\delta_{0}$. In (c) and (d), the time $\widehat{t}$ is scaled by $\delta_{0}^{2} / D_{\mathrm{MB}}^{\mathrm{o}}$, and thickness $\widehat{\delta}$ is scaled by $\delta_{0}$.

Figure 6 shows the transient displacement of the liquid-vapor interface based on the long-time scale. The asymptotic result is consistent with the numerical model. The overall evaporation rate and thus the interfacial moving speed gradually slows down until methanol is fully depleted. In contrast to the short-time limit, the deviation between the analytical approximation and numerical result increases as the partition number $\gamma$ decreases. In the long-time approximation, variables $D^{(\ell)}, \mathrm{M}^{(\ell)}, \rho^{(\ell)}$ and $\rho^{(\mathrm{g})}$ are assumed constant. The 
evaporation kinetics is dominated by methanol diffusion in the liquid domain and the concentration in the gas phase almost reaches equilibrium. In the intermediate time regime only numerical result is available for the comparison with experimental data, which will be shown later in Fig. 9.

The scaling analysis in section II manifests that evaporation is controlled by five dimensionless parameters: the diffusivity ratio $\alpha$, the partition number $\gamma$, the density ratio $\lambda$, the dimensionless total length $\widehat{b}$, and the initial methanol mass fraction $\omega_{\mathrm{M}}^{\mathrm{o}}$. Among these five parameters, $\widehat{b}$ and $\omega_{\mathrm{M}}^{\mathrm{o}}$ depend on the sample size and initial condition, and $\lambda$ is related to the initial densities of liquid and gas (air). These three parameters do not provide much information about the transient behavior of each component in the liquid mixture during evaporation. The two parameters that reveal the impact of volatile component on the evaporation are $\alpha$ and $\gamma$, where $\alpha$ represents the transport ability of volatile component in both phases, and $\gamma$ determines the concentration jump at the interface. The raise of $\gamma$ indicates more methanol vapor could stay at the surface, which provides a large concentration gradient to enhance the evaporation rate. When $\gamma \rightarrow 0$, no methanol vapor is stored at the interface and evaporation vanishes.

Figure 7 demonstrates the scaled mass concentration of methanol in both liquid and gas phases in the short- $(\mathrm{a}, \mathrm{b})$ and long-time $(\mathrm{c}, \mathrm{d})$ regimes using the transform spatial coordinate $\eta$. The solid lines are numerical results; the dashed lines and circles are analytical approximations. Note that the mass concentrations $\rho_{\mathrm{M}}^{(\ell)}$ and $\rho_{\mathrm{M}}^{(\mathrm{g})}$ are scaled differently in liquid and gas phases. Within the short-time limit, the concentration in liquid drops off slightly $(<0.02)$ near the interface while the concentration in the rest of the liquid domain remains unchanged. In the gas phase, the diffusive wave propagates quickly into the gas domain. An equilibrium profile is almost achieved as the scaled time reaches 0.5. The fast transient behavior is understandable because the molecular diffusivity in the gas phase is about four to five orders of magnitude larger than that in the liquid phase. The small concentration drops down $1.5 \%$ at the liquid-vapor interface shown in Fig. $7 \mathrm{~b}$ in order to satisfy the partition condition. The simplified analytical approximation in general very well predicts the transient behaviors in the gas phase. Although a constant bulk concentration is applied in the liquid phase, the partition condition is still preserved in the approximation. In the long-time regime, shown in Figs. $7 \mathrm{c}$ and $7 \mathrm{~d}$, obviously the evaporation kinetics is limited by diffusion in the liquid domain, and almost all concentration profiles shown in the 
gas phase are fully relaxed at the selected time instants. Again, this is because diffusivity in gas phase is much larger than diffusivity in liquid. As expected the asymptotic result overlaps with numerical results completely at longer times. The concentration profile, and thus the corresponding mass flux, decays continuously until methanol is fully evaporated.

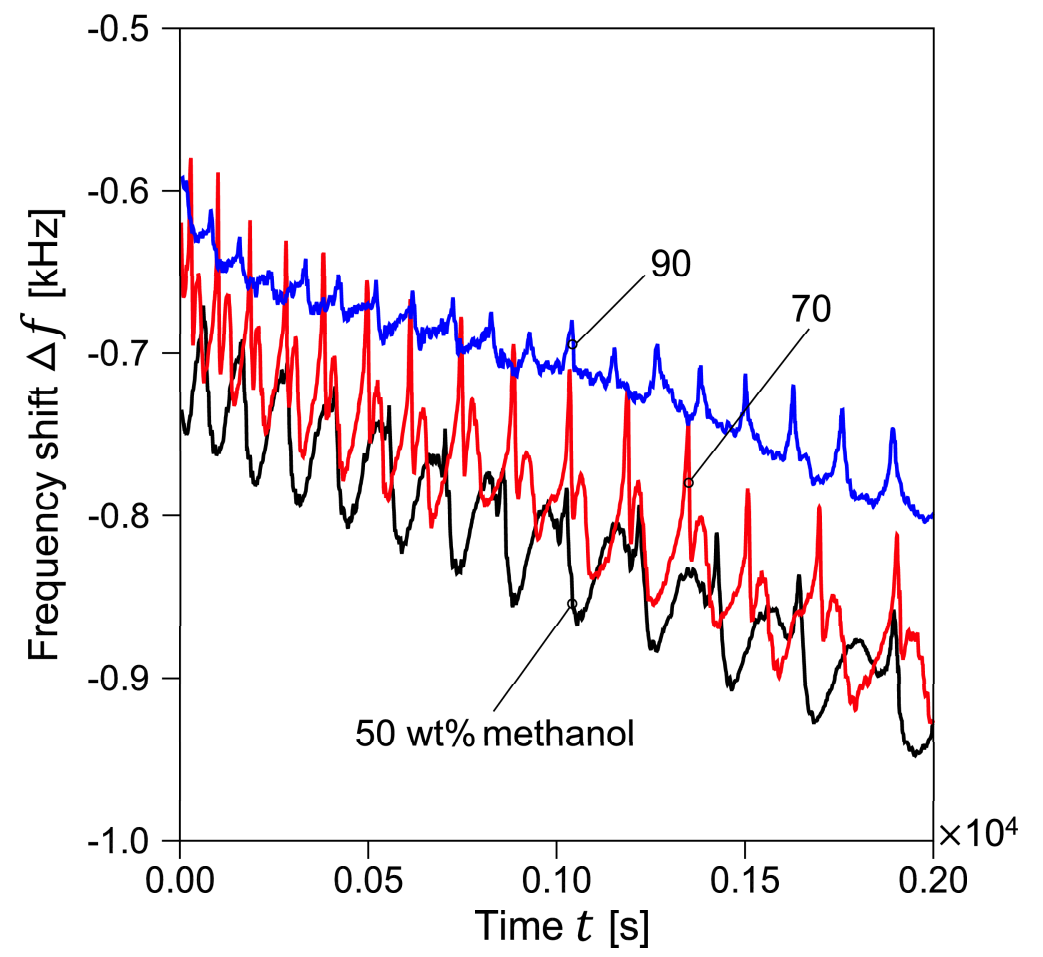

FIG. 8: Shift of the resonant frequency versus time for a crystal resonator loaded with methanolbutanol mixture. Initially the liquid mixture contains 50, 70, or $90 \mathrm{wt} \%$ of methanol. About $1 \mathrm{~mL}$ of the mixture is deployed on top of the crystal surface under ambient conditions.

Figure 8 provides the raw experimental data, representing the shift of the resonant frequency $\triangle f$ versus time for a loaded crystal resonator during the early stage of the evaporation experiment. The reduction of the resonant frequency is highly correlated to the methanol weight percentage. The evaporation kinetics is determined by the time difference between spikes, while the change of viscosity of the mixture at $\eta \simeq 0$ is approximated by a base line connecting the foot regions of the continuous spikes. Overall, the reduction curve drops significantly at the beginning stage, consistent with the model prediction. The mass densities of methanol and butanol are close to each other, but the butanol viscosity is about an order of magnitude higher than methanol, and thus the overall reduction is primarily contributed by the raise of viscosity of the liquid mixture due to the fast evaporation of 
methanol and the increase of butanol mole fraction. After the initial drop, the reduction of resonant frequency levels off, indicating that methanol has almost depleted and most of the butanol remains. The spike pattern continues until near the end of evaporation, where the signal becomes noisy due to the exposure of bare crystal and the formation of moving contact line around the remaining liquid film.

Figure 9 shows the comparison of experimental and modeling results of the transient evolution of the moving liquid-vapor interface (a) and effective viscosity (b) at three initial mass fractions of methanol in the liquid mixture. The experimental data is extracted from the frequency response given in Fig. 8. As shown in Fig. 9a, overall the numerical model has well predicted the experimental results for cases with relatively high initial methanol mass fractions. The interfacial moving velocity is on the order of $1 \mu \mathrm{m} / \mathrm{s}$. The deviation increases as methanol mass fraction reduces, more likely due to our simplification on the assumed non-volatile butanol. In practice, 1-butanol also evaporates into the gas phase with a smaller evaporation pressure. This is why the data points continue to drop while in the model prediction the evaporation curve levels off when methanol is almost depleted. The transition of fast to slow evaporation process is clearly shown by the experimental data. Also in the intermediate time, approximately $\sim 10^{3}$ to $5 \times 10^{3} \mathrm{~s}$, the analytical approximation is no longer applicable to represent the numerical result. Figure $9 \mathrm{~b}$ presents extracted viscosity of liquid mixture near the top surface of the crystal resonator based on Eq. (53). The mass concentration in the liquid phase varies but the mass density is approximately a constant. For the model prediction, the effective viscosity at $z \simeq 0$ can be obtained by the local mole fractions or mass concentration of the binary mixture [40], expressed as

$$
\ln \eta_{\mathrm{L}}=x_{\mathrm{M}} \ln \eta_{\mathrm{M}}+x_{\mathrm{B}} \ln \eta_{\mathrm{B}}
$$

where $\eta_{\mathrm{L}}$ is the viscosity of the mixture, $\eta_{\mathrm{M}}$ and $\eta_{\mathrm{B}}$ are the known viscosities of pure methanol and 1-butanol, respectively, and $x_{\mathrm{M}}$ and $x_{\mathrm{B}}$ are the corresponding local mole fractions. The viscosities of pure methanol and pure butanol provide the lower and upper bounds (dashed lines) for the effective viscosity of the mixture. During evaporation, the effective viscosity increases and approaches butanol's viscosity when the amount of methanol reduces. However, it is not yet clear to us why at lower percentage of methanol the viscosity does not recover to 1-butanol's viscosity. Calibrations have shown that the instrumental uncertainty on viscosity measurement of aqueous solution of glycerol with various concentration has 

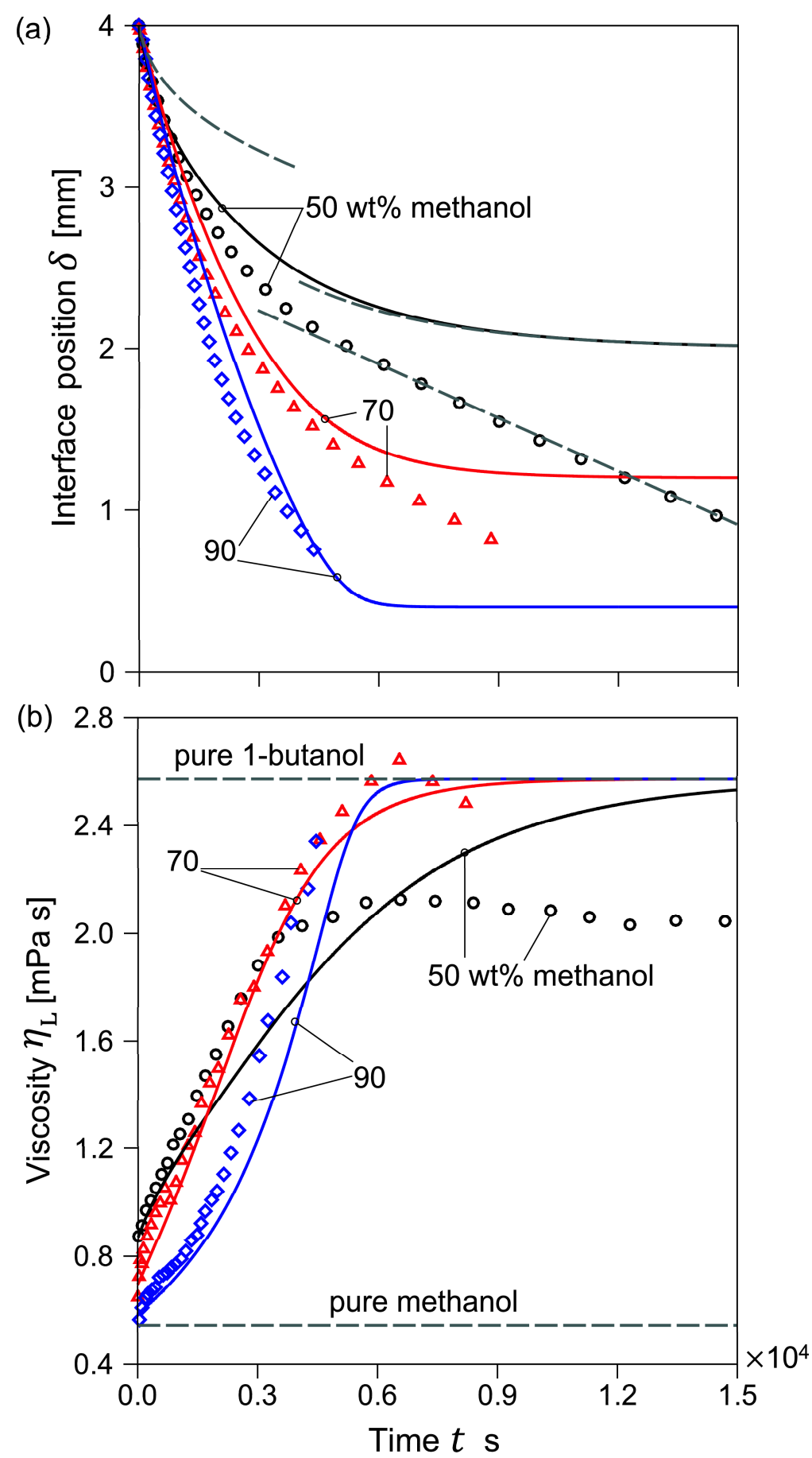

FIG. 9: (a) Transient evolution of the liquid-vapor interface under various methanol mass fractions. The experimental data (symbols) are compared with numerical results (solid lines). The analytical approximations (dashed lines) in the short- and long-time limits are provided for the case of $50 \mathrm{wt} \%$. All parameters used are listed in Table I. (b) The corresponding effective viscosity located at the bottom of the liquid mixture. 
about $10 \%$ relative error. Furthermore, in the long-time regime, the experimental data have a non-monotonic increase of viscosity. This may be due to the competing effects of evaporating cooling and heat transfer from the substrate.

To better justify the theoretical and experimental uncertainties, we consider the following thermal energy equation for the temperature distribution within the liquid domain and a quasi-steady interfacial condition based on local energy balance:

$$
\frac{1}{\mathcal{F}_{\mathrm{o}}} \frac{\partial T^{(\ell)}(0 \leq z \leq \delta)}{\partial t}=\frac{\partial^{2} T^{(\ell)}}{\partial z^{2}}+\mathcal{B}_{\mathrm{r}}\left(\frac{\partial u}{\partial z}\right)^{2}, \quad \frac{\partial T^{(\ell)}(z=\delta)}{\partial z} \simeq E_{\mathrm{c}} \frac{\partial \rho_{\mathrm{M}}^{(g)}}{\partial z}
$$

where the Fourier number $\mathcal{F}_{\mathrm{o}}=\tau \alpha^{(\ell)} / \delta^{2}$, Brinkman number $\mathcal{B}_{\mathrm{r}}=\mu^{(\ell)} U^{2} /\left(\kappa^{(\ell)} \triangle T^{(\ell)}\right)$, and the Evaporation cooling number [41] $E_{\mathrm{c}}=L_{\mathrm{v}} D^{(g)} c_{1} / \kappa^{(\ell)}$. The parameters $T^{(\ell)}$ and $u$ are scaled temperature and velocity fields, respectively, and $\rho_{\mathrm{M}}^{(g)}$ is the scaled methanol vapor mass concentration at the interface. Properties $\kappa^{(\ell)}, \alpha^{(\ell)}, \mu^{(\ell)}, L_{\mathrm{v}}$, and $D^{(g)}$ are thermal conductivity, thermal diffusivity, dynamic viscosity, latent heat for vaporization, and vapor diffusivity, respectively. $\tau$ can be the characteristic short- or long-time scales, $\delta$ is the characteristic thickness of the liquid layer, $U$ is characteristic velocity, and constant $c_{1}$ is from the linear approximation of the increase of saturation vapor concentration per kelvin. In the long-time regime, $\tau \sim 10^{4} \mathrm{~s}, \mathcal{F}_{\mathrm{o}}$ for either methanol or butanol is on the order of $10^{2}$, and thus the thermal energy equation is approximately quasi-steady and the temperature increase due to viscous dissipation should correspond to $\mathcal{B}_{\mathrm{r}} \sim 1$. For a displacement of 1 to $2 \mathrm{~nm}$ at the center of the crystal at $\sim 5 \mathrm{MHz}$ resonance frequency, the characteristic fluid velocity is about 3 to $5 \mathrm{~cm} / \mathrm{s}$. The viscosity of methanol and butanol at room temperature are given in Table I, the thermal conductivity of methanol and butanol are about 0.15 to $0.20 \mathrm{~W} / \mathrm{mK}$ at room temperature, and thus under steady state condition the characteristic temperature increase is merely $10^{-6}$ to $10^{-5} \mathrm{~K}$. Therefore, the heating effect due to viscous dissipation is indeed negligible. For the cooling effect caused by methanol evaporation, the latent heat $L_{\mathrm{v}}$ is around $1.2 \times 10^{6} \mathrm{~J} / \mathrm{kg}$, gas diffusivity is $1.5 \times 10^{-5} \mathrm{~m}^{2} / \mathrm{s}, c_{1} \sim 9.5 \times 10^{-3} \mathrm{~kg} / \mathrm{m}^{3} \mathrm{~K}$, and thus the evaporative cooling number $E_{\mathrm{c}}$ is about 1.14. Since $E_{\mathrm{c}}$ is around 1, which enters the regime that evaporation cooling may considerably reduce the saturation pressure and thus reduce the evaporative flux. According to the studies of evaporative cooling effect on a sessile methanol droplet, the range of temperature difference within the droplet is about 2 degree kelvin [42], which corresponds to $\sim 10$ to $15 \%$ reduction of evaporative flux [41], and $\sim 3 \%$ increase of the local viscosity. By neglecting evaporative cooling, our modeling results would 
have over-predicted (instead of under-predicted) the evaporation rate. Therefore, the fact that modeling results have under-predicted the evaporative rate (modeling has slower moving interface) is not due to evaporative cooling but more likely due to the neglected butanol evaporation. Systems involving two or multiple volatile components are more challenging in determining the partition condition at the interface and mutual diffusion coefficients in the gas phase. Besides, the contribution of secondary flow to the resistance impedance may become significant as the liquid film becomes thinner. These complicated issues, along with the coupling of heat and mass transfer are to be investigated in future studies.

\section{CONCLUSION}

Investigation on the evaporation kinetics and fluid properties of binary mixtures using quartz crystal resonator is demonstrated under ambient condition. Selected light monohydric alcohols are used because of their high volatility and ideal solution behavior. A numerical model and analytical approximation are developed to interpret the frequency response of the resonator loaded with the liquid mixture. Precision measurement of the evaporation kinetics of the liquid layer is evaluated by the spikes created by the longitudinal wave, while the viscosity stratification due to the concentrated butanol is evaluated by the shear wave. The evaporation flux can therefore be characterized by the velocity of the moving liquidvapor interface. Future work will consider evaporation kinetics of a mixture of many volatile components.

\section{ACKNOWLEDGMENTS}

Acknowledgment is made to the donors of the American Chemical Society Petroleum Research Fund for the support of this research. H. Song, C. Basdeo, and T.-H. Fan also acknowledge the support from NSF CMMI-0952646.

[1] E. Bonaccurso and H.-J. Butt, Microdrops on atomic force microscope cantilevers: evaporation of water and spring constant calibration, J. Phys. Chem. B, 2005, 109, 253-263. 
[2] W.P. Mason, W.O. Baker, H.J. McSkimin, and J.H. Heiss, Measurement of shear elasticity and viscosity of liquids at ultrasonic frequencies, Phys. Rev., 1949, 75(6), 936-946.

[3] M. Rodahl and B. Kasemo, A simple setup to simultaneously measure the resonant frequency and the absolute dissipation factor of a quartz crystal microbalance, Rev. Sci. Instrum., 1996, 67(9), 3238-3241.

[4] A. Saluja and D.S. Kalonia, Measurement of fluid viscosity at microliter volumes using quartz impedance analysis, AAPS PharmSciTech, 2004, 5(3), 68-81.

[5] J. Zhang, X.D. Su, and S.J. O'shea, Antibody/antigen affinity behavior in liquid environment with electrical impedance analysis of quartz crystal microbalances, Biophys. Chem., 2002, $99(1), 31-41$.

[6] D. Johannsmann, Viscoelastic, mechanical, and dielectric measurements on complex samples with the quartz crystal microbalance, Phys. Chem. Chem. Phys., 2008, 10(31), 4516-4534.

[7] M.J. Joyce, P. Todaro, R. Penfold, S.N. Port, J.A.W. May, C. Barnes, and A.J. Peyton, Evaporation of sessile drops: Application of the quartz crystal microbalance, Langmuir, 2000, 16(8), 4024-4033.

[8] L. McKenna, M.I. Newton, G. McHale, R. Lucklum, and J. Schroeder, Compressional acoustic wave generation in microdroplets of water in contact with quartz crystal resonators, J. Appl. Phys., 2001, 89(1), 676-680.

[9] G. Couturier, R. Boisgard, C. Jai, and J.P. Aimé, Compressional wave generation in droplets of water deposited on a quartz crystal: Experimental results and numerical calculations, $J$. Appl. Phys., 2007, 101(9), 093510.

[10] H. Zhuang, P. Lu, S.P. Lim, and H.P. Lee, Study of the evaporation of colloidal suspension droplets with the quartz crystal microbalance, Langmuir, 2008, 24(15), 8373-8378.

[11] N.T. Pham, G. McHale, M.I. Newton, B.J. Carroll, and S.M. Rowan, Application of the quartz crystal microbalance to the evaporation of colloidal suspension droplets, Langmuir, 2004, 20(3), 841-847.

[12] F.N. Nunalee, K.R. Shull, B.P. Lee, and P.B. Messersmith, Quartz crystal microbalance studies of polymer gels and solutions in liquid environments, Anal. Chem., 2006, 78(4), 1158-1166.

[13] G.Z. Sauerbrey, Use of quartz vibration for weighing thin films on a microbalance, J. Physik , 1959, 155, 206-212.

[14] H. Zhuang, P. Lu, S.P. Lim, and H.P. Lee, Frequency response of a quartz crystal microbalance 
loaded by liquid drops, Langmuir, 2007, 23(13), 7392-7397.

[15] K.K. Kanazawa and J.G. Gordon, The oscillation frequency of a quartz resonator in contact with a liquid, Anal. Chim. Acta, 1985, 175, 99-105.

[16] K.K. Kanazawa and J.G. Gordon, Frequency of a quartz microbalance in contact with liquid, Anal. Chem., 1985, 57(8), 1770-1771.

[17] Z. Lin and M.D. Ward, The role of longitudinal waves in quartz crystal microbalance applications in liquids, Anal. Chem., 1995, 67(4), 685-693.

[18] B.A. Martin and H.E. Hager, Flow profile above a quartz crystal vibrating in liquid, J. Appl. Phys., 1989, 65(7), 2627-2629.

[19] J. Stefan, Über die Verdampfung und die Aufösung als Vorgange der Diffusion, Ann. Phys. (Berlin), 1890, 277(12), 725-747.

[20] C. Vuik, Some historical notes about the Stefan problem, Delft University of Technology, Faculty of Technical Mathematics and Informatics, 1993.

[21] C.Y. Lee and C.R. Wilke, Measurements of vapor diffusion coefficient, Ind. Eng. Chem., 1954, 46(11), 2381-2387.

[22] A.T. Prata and E.M. Sparrow, Diffusion driven nonisothermal evaporation, J. Heat Transfer., 1985, 107(1), 239-242.

[23] J.C. Slattery and V.R. Mhetar, Unsteady-state evaporation and the measurement of a binary diffusion coefficient, Chem. Eng. Sci., 1997, 52(9), 1511-1515.

[24] V.R. Mhetar and J.C. Slattery, The Stefan problem of a binary liquid mixture, Chem. Eng. Sci., 1997, 52(8), 1237-1242.

[25] R. Chebbi and M.S. Selim, The Stefan problem of evaporation of a volatile component from a binary liquid mixture, Heat Mass Transf., 2006, 42(3), 238-247.

[26] R. Carty and T. Schrodt, Concentration profiles in ternary gaseous diffusion, Ind. Eng. Chem. Fund., 1975, 14(3), 276-278.

[27] J.F. Richardson, Evaporation of two-component mixtures, Chem. Eng. Sci., 1959, 10(4), 234242 .

[28] R.D. Goodwin, Methanol thermodynamic properties from 176 to $673 \mathrm{~K}$ at pressures to 700 bar, J. Phys. Chem. Ref. Data, 1987, 16(4), 799-892.

[29] H.R. Kemme and S.I. Kreps Vapor pressure of primary n-alkyl chlorides and alcohols, $J$. Chem. Eng. Data, 1969, 14(1), 98-102. 
[30] A. Vignes, Diffusion in binary solutions: variation of diffusion coefficient with composition, Ind. Eng. Chem. Fund., 1966, 5(2), 189-199.

[31] F.O. Shuck and H.L. Toor, Diffusion in the three component liquid system methyl alcohol-npropyl alcohol-isobutyl alcohol, J. Phys. Chem., 1963, 67(3), 540-545.

[32] B. González, N. Calvar, E. Gómez, and Á. Domínguez, Density, dynamic viscosity, and derived properties of binary mixtures of methanol or ethanol with water, ethyl acetate, and methyl acetate at $\mathrm{T}=(293.15,298.15$, and 303.15) K, J. Chem. Thermodyn., 2007, 39(12), 1578-1588.

[33] G.P. Dubey, M. Sharma, and N. Dubey, Study of densities, viscosities, and speeds of sound of binary liquid mixtures of butan-1-ol with n-alkanes (C 6, C 8, and C 10) at T=(298.15, 303.15, and 308.15) K, J. Chem. Thermodyn., 2008, 40(2), 309-320.

[34] S.M. Reddy, J.P. Jones, and T.J. Lewis, The coexistence of pressure waves in the operation of quartz-crystal shear-wave sensors, J. Appl. Phys., 1998, 83(5), 2524-2532.

[35] T.W. Schneider and S.J. Martin, Influence of compressional wave generation on thicknessshear mode resonator response in a fluid, Anal. Chem., 1995, 67(18), 3324-3335.

[36] S.M. Pereira, M.A. Rivas, and T.P. Iglesias, Speeds of sound, densities, isentropic compressibilities of the system (methanol+ polyethylene glycol dimethyl ether 250) at temperatures from 293.15 to 333.15 K, J. Chem. Thermodyn., 2003, 35(3), 383-391.

[37] M. Domínguez, C. Lafuente, M.C. López, F.M. Royo, and J.S. Urieta, Speed of sound and isentropic compressibility of (1-butanol+ n-hexane+ 1-chlorobutane) and the constituent binary mixtures at the temperatures $298.15 \mathrm{~K}$ and $313.15 \mathrm{~K}$, J. Chem. Thermodyn., 2000, 32(2), $155-173$.

[38] C. Pozrikidis, Introduction to Theoretical and Computational Fluid Dynamics, Oxford University Press, London, 1997.

[39] W.H. Press, S.A. Teukolsky, W.T. Vetterling, and B.P. Flannery, Numerical Recipes in C: The Art of Scientific Computing, Cambridge University, New York, 1992.

[40] V.A. Bloomfield and R.K. Dewan, Viscosity of liquid mixtures, J. Phys. Chem., 1971, 75(20), 3113-3119.

[41] X. Xu, L. Ma, Analysis of the effects of evaporative cooling on the evaporation of liquid droplets using a combined field approach, Scientific Reports, 2015, 5(8614), DOI:10.1038/srep08614.

[42] G.J. Dunn, S.K. Wilson, B.R. Duffy, S. David, K. Sefiane, The strong influence of substrate conductivity on droplet evaporation, Journal of Fluid Mechanics, 2009, 623, 329-351. 